\title{
Phase Synchrony among Neuronal Oscillations in the Human Cortex
}

\author{
J. Matias Palva, ${ }^{1}$ Satu Palva, ${ }^{2,3}$ and Kai Kaila ${ }^{1}$ \\ ${ }^{1}$ Department of Biological and Environmental Sciences and ${ }^{2}$ Cognitive Brain Research Unit, Department of Psychology, University of Helsinki, FIN-00014 \\ Helsinki, Finland, and ${ }^{3}$ BioMag Laboratory, Helsinki University Central Hospital, FIN-00029 Helsinki, Finland
}

Synchronization of neuronal activity, often associated with network oscillations, is thought to provide a means for integrating anatomically distributed processing in the brain. Neuronal processing, however, involves simultaneous oscillations in various frequency bands. The mechanisms involved in the integration of such spectrally distributed processing have remained enigmatic. We demonstrate, using magnetoencephalography, that robust cross-frequency phase synchrony is present in the human cortex among oscillations with frequencies from 3 to $80 \mathrm{~Hz}$. Continuous mental arithmetic tasks demanding the retention and summation of items in the working memory enhanced the cross-frequency phase synchrony among $\alpha(\sim 10 \mathrm{~Hz}), \beta(\sim 20 \mathrm{~Hz})$, and $\gamma(\sim 30-40 \mathrm{~Hz})$ oscillations. These tasks also enhanced the "classical" within-frequency synchrony in these frequency bands, but the spatial patterns of $\alpha, \beta$, and $\gamma$ synchronies were distinct and, furthermore, separate from the patterns of cross-frequency phase synchrony. Interestingly, an increase in task load resulted in an enhancement of phase synchrony that was most prominent between $\gamma$ - and $\alpha$-band oscillations. These data indicate that crossfrequency phase synchrony is a salient characteristic of ongoing activity in the human cortex and that it is modulated by cognitive task demands. The enhancement of cross-frequency phase synchrony among functionally and spatially distinct networks during mental arithmetic tasks posits it as a candidate mechanism for the integration of spectrally distributed processing.

Key words: $\alpha$; attention; cortex; $\gamma$; oscillator; parietal; synchrony; working memory

\section{Introduction}

A major challenge in neuroscience has been to elucidate the mechanisms that integrate spatially distributed processing and signal the relatedness of neurons coding different features of the same object (Singer, 1999). This "binding problem" is thought to be solved by synchronization of neurons into transient oscillatory assemblies (Singer, 1999; Varela et al., 2001). Indeed, synchronous oscillations in $\beta$-frequency $(14-25 \mathrm{~Hz})$ and $\gamma$-frequency $(25-70 \mathrm{~Hz}$ ) bands have been implicated in feature binding (Gray et al., 1989; Singer, 1999; Tallon-Baudry and Bertrand, 1999; Varela et al., 2001), and they have also been detected during working memory maintenance when sustained activation of neural representations is required (Tallon-Baudry et al., 2001; Pesaran et al., 2002). Therefore, it has been concluded that these oscillations may represent the contents of working memory. In humans, $\theta$-band (4-7 Hz) (Raghavachari et al., 2001; Halgren et al., 2002) and $\alpha$-band (7-14 Hz) (Halgren et al., 2002; Jensen et al., 2002) oscillations are also linked to working memory. However, they do not appear to underlie feature integration per se (Singer, 1999; Howard et al., 2003) but are instead involved in

Received 0ct. 13, 2004; revised March 4, 2005; accepted March 4, 2005.

This work was supported by the Academy of Finland, the Sigrid Juselius Foundation, the Foundation for the Advancement of Technology, the Oskar Öflund Foundation, and the Ella and Georg Ehrnrooth Foundation. We are grateful to Gábor Tamás, Christopher Bailey, and Sampsa Vanhatalo for helpful comments on previous versions of this paper.

Correspondence should be addressed to J. Matias Palva, Department of Biological and Environmental Sciences, P.0. Box 65 (Viikinkaari 1), University of Helsinki, FIN-00014 Helsinki, Finland. E-mail: matias.palva@helsinki.fi. DOI:10.1523/JNEUROSCI.4250-04.2005

Copyright $\odot 2005$ Society for Neuroscience $\quad$ 0270-6474/05/253962-11\$15.00/0 top-down modulation (von Stein et al., 2000; Fanselow et al., 2001; Mima et al., 2001). Hence, in contrast to the contentmediating $\beta$ - $/ \gamma$-band oscillations, the $\theta-/ \alpha$-band oscillations serve top-down modulating, "attentional," and other contextdefining (Llinás et al., 1998) functions. Thus, there is the problem of how the various assemblies at different levels of functional hierarchy (cf. Hochstein and Ahissar, 2002) act in concert to support unified cognitive functions. Evidently, this demands dynamic interactions between activities in distinct frequency bands (Engel and Singer, 2001; VanRullen and Koch, 2003), but the nature of these interactions has remained empirically unresolved.

Bispectral measures such as bicoherence (Dumermuth et al., 1971; Jeffrey and Chamoun, 1994) have been used to detect nonlinear cross-frequency (CF) phase coupling in the cat cortex (Schanze and Eckhorn, 1997; von Stein et al., 2000) and in human electroencephalograms (EEGs) (Jeffrey and Chamoun, 1994; Shils et al., 1996; Schack et al., 2002). However, like conventional coherence (cf. Tass et al., 1998), bicoherence mixes phase and amplitude information. Hence, it leaves the specific form and, consequently, also the putative neuronal mechanisms of the phase coupling unclear. To date, two principal forms of CF phase interactions have been recognized: $n: m$ phase synchrony, which indicates amplitude-independent phase locking of $n$ cycles of one oscillation to $m$ cycles of another oscillation (Tass et al., 1998), and nested oscillations, which reflect the locking of the amplitude fluctuations of faster oscillations to the phase of a slower oscillation (Vanhatalo et al., 2004). Although there is evidence for nested oscillations in the human brain (Schack et al., 2002; Bruns 
Aa

$1.70 \mathrm{~Hz}$ Whandwh

$8-16 \mathrm{~Hz}$

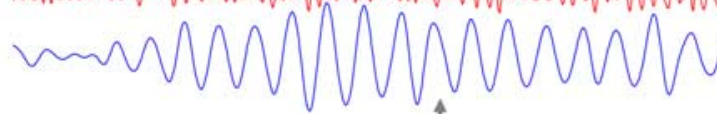

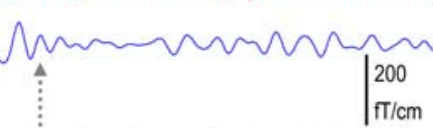

B

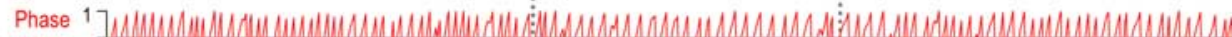
$24-50 \mathrm{~Hz}_{-1}$ ב Phase ${ }^{1} 16 \mathrm{~Hz}$

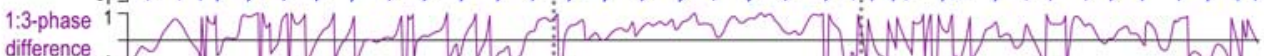

c

D

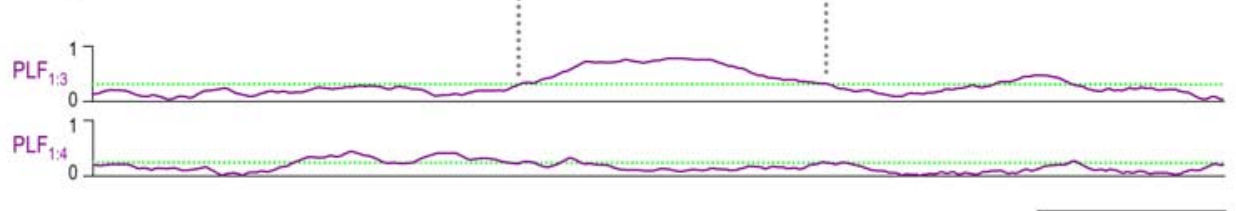

$500 \mathrm{~ms}$
Ab

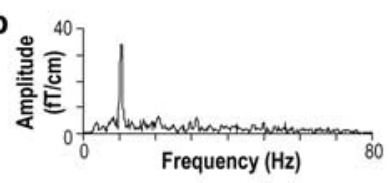

E
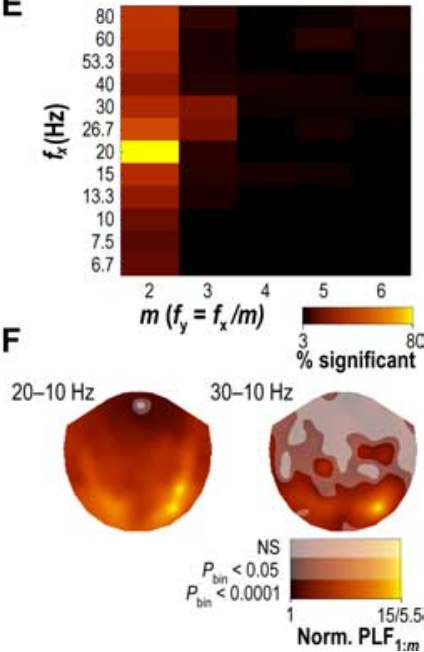

Figure 1. Local CF phase synchrony in the human cortex recorded with magnetoencephalography. Aa, The gradiometer signal (black) over the left occipitoparietal region shows a burst of $\sim 10$ $\mathrm{Hz}$ oscillations (blue) and intermittent $30-40 \mathrm{~Hz}$ oscillations (red). $\boldsymbol{A} \boldsymbol{b}$, Amplitude spectrum of the gradiometer signal in $\boldsymbol{A a}$. $\boldsymbol{B}, 0$ scillation phases (red and blue; normalized with $1 / \boldsymbol{\pi}$ ) and their $1: 3$ phase difference (purple). C, 1:3 Phase synchrony, quantified in sliding windows of $500 \mathrm{~ms}$. The green line indicates $95 \%$ confidence limits. The gray arrows mark a period of prominent synchrony. D, 1:3 Phase synchrony occurred intermittently with 1:3 phase synchrony. $\boldsymbol{E}, 1: \mathrm{m}$ Phase synchrony mapped with Morlet wavelets over the 20 min recording sessions. The color scale indicates the percentage of gradiometers detecting statistically significant $(p<0.01)$ synchrony between oscillations at frequencies $f_{x}$ and $f_{y}\left(f_{y}=f_{x} / m\right)$ averaged over subjects $(N=17)$. The lower end of the color scale is set by the binomial probability ( $p_{\text {bin }}$ ) that is $<0.02$ for finding by chance $>3 \%$ of the gradiometers to show statistically significant synchrony. $F$, Topographies of $\beta-\alpha$ (left) and $\gamma-\alpha$ (right) phase synchronies. The color scale indicates the normalized (Norm.) phase-locking factor, averaged over subjects. The levels of transparency indicate $p_{\text {bin }}$ for finding by chance the proportion of subjects with a statistically significant synchrony [note that $p_{\mathrm{bin}}<0.0001$ corresponds to $p_{\mathrm{bin}, \mathrm{B}}<0.01$ with Bonferroni's correction for the number of gradiometers (102)]. NS, Not significant. The MEG helmet covering the whole scalp is flattened, and anterior direction is upward.

and Eckhorn, 2004; Vanhatalo et al., 2004) and in the rat hippocampus (Chrobak and Buzsaki, 1998), n:m phase synchrony has not been observed among cortical oscillations (cf. Tass et al., 1998, 2003). $n: m$ phase synchrony is, nevertheless, an attractive means for achieving CF integration, because, unlike nested oscillations, it operates with the temporal precision of the faster oscillation and hence conserves the temporal codes therein.

In the present work, we used neuromagnetic recordings to investigate whether the human cortex exhibits CF $(n: m)$ phase synchrony among ongoing oscillations. We then used continuous mental arithmetic tasks to ask whether cognitive operations are associated with changes in CF phase synchrony and to examine the notion that the contents-to-context binding involves phase synchrony between the constituent $\beta$ - $/ \gamma$-band and $\theta-/ \alpha$ band oscillations.

\section{Materials and Methods}

Recordings and tasks. We recorded data from 17 healthy subjects using a whole-scalp magnetoencephalography (MEG) system with 204 gradiometer and 102 magnetometer channels (Elekta Neuromag, Helsinki, Finland). In all sessions, the subjects sat relaxed with their eyes closed in a dimly lit and acoustically insulated room and performed one of three uninterrupted mental tasks in a pseudorandom order, each lasting 20 min. The subject's head position in the MEG system was identical during all tasks with an accuracy of $\sim 3 \mathrm{~mm}$, measured using three coils attached to the head. The recorded data were high- and low-pass filtered on-line at 0.03 and $100 \mathrm{~Hz}$, respectively, sampled at $600 \mathrm{~Hz}$, and decimated off-line to a sampling rate of $300 \mathrm{~Hz}$.

In one session, the subjects were instructed to actively clear their imagined visual and auditory fields [active rest (R)]. During the other two tasks [task 1 (T1) and T2], they were instructed to visualize two or three numbers, respectively, that were given by the experimenter at the onset of task execution, calculate their sum, discard the first of the original numbers, and place the sum as the last number. This was iterated until the sum exceeded 100, after which the last two or three digits of the final sum were used as a new set of starting numbers (see Fig. $2 A$ ). The task R thus minimized the number of simultaneously active neural representations as well as the multitude of cognitive processes requiring integration. T1 and $\mathrm{T} 2$, however, required the working memory system to operate near or at its $3 \pm 1$ object capacity limit (Luck and Vogel 1997) and also demanded the coordination of several cognitive operations including, for example, the simultaneous maintenance of several neural number representations and their arithmetical summation. Together, the comparison of $\mathrm{R}$ with $\mathrm{T} 1 / \mathrm{T} 2$ reflects the gross difference between active rest and active cognitive processing, whereas the comparison between $\mathrm{T} 1$ and $\mathrm{T} 2$ reveals processes associated with increasing working memory load. The subjects were interviewed after the recordings, and they rated the task difficulty and subjective success in task performance. All tasks were reported to be difficult, and all demanded a highly attentive and aroused state.

Continuous working memory tasks (e.g., of $n$-back type) have been used previously in EEG (Gevins et al., 1997) and imaging (Carlson et al., 1998) studies. Unlike the $n$-back tasks, ours did not use external stimuli to avoid the complications of extensive stimulus-locked oscillations (Lachaux et al., 1999; Halgren et al., 2002). An obvious drawback of continuous tasks is that they do not allow the dissection of encoding, retention, and retrieval processes of working memory or, in our case, the dissection of working memory-specific processes from those underlying the mental arithmetic. Nevertheless, in continuous tasks, the lack of the temporal dimension greatly simplifies both the data analysis and visualization.

Wavelet filtering. The amplitude and phase of ongoing oscillations were obtained as a function of time using Morlet wavelets. Convolution $X\left(t, f_{0}\right)=x(t) \times w\left(t, f_{0}\right)$ of the signal $x(t)$ with the Morlet wavelet $w\left(t, f_{0}\right)=$ $A_{\exp }\left(-t^{2} / 2 \sigma_{t}^{2}\right) \exp \left(2 i \pi f_{0} t\right)$, where $\sigma_{t}=m^{\prime} / 2 \pi f_{0}$ and $i$ is the imaginary 
unit, gives a complex vector $X\left(t, f_{0}\right)$ of which the real part corresponds to the filtered signal $x$, modulus corresponds to the amplitude envelope, and angle corresponds to the phase of the signal $x$ in a frequency band with a center frequency of $f_{0}$ and a frequency-domain SD of $\sigma_{f}=f_{0} / m^{\prime}$. The constant $m^{\prime}$ defines the compromise between time and frequency precision. The results shown in this study were essentially identical with values of $m^{\prime}$ ranging from 6.0 to 8.5 .

Narrow time-window filtering. Because of their Gaussian shape in both time and frequency domains, Morlet wavelets are limited to $m^{\prime}$ values $>5$, which sets the minimum time-domain $\mathrm{SD}\left(\sigma_{t}\right)$ to $\sim 0.8 \mathrm{~s}$ for unit frequency. To obtain filter families with much less time-domain spread, we used combinations of high- and low-pass finite-impulse response (FIR) filters that were characterized by high-pass stop bands of 0.3 and lowpass stop bands of 3.0 for a center frequency of 1.0 (giving $3 \mathrm{~dB}$ attenuation at 0.8 and 1.65 , respectively). The time-domain SD for these filters is $\sim 0.4 \mathrm{~s}$ for unit frequency. When using FIR filters, the imaginary part for the complex vector $X(t, f)$ was obtained with the Hilbert transform. These filters were used in Figures $1 A-D$ and $3, A$ and $C$.

Phase synchrony. Oscillations at frequencies $f_{x}$ and $f_{y}$, of which the ratio is given by the integers $n$ and $m$ so that $n f_{x}=m f_{y}$, may be considered $n: m$ phase synchronized when their phase difference $\left(\omega_{n, m}, \omega_{n, m}=n \theta_{x}-\right.$ $m \theta_{y}$, where $\theta_{x}$ and $\theta_{y}$ are the phases of oscillations $x$ and $\left.y\right)$ is not uniformly distributed (Tass et al., 1998). We evaluated the presence of nonrandom phase ordering using the phase-locking factor (PLF) for frequency ratios with $n=1$ and $m=1-6$. For $N$ complex numbers $z_{i}, i=$ 1 ... N, with unit modulus, the PLF is given by $N^{-1} \Sigma z_{i}$.

Statistical evaluation of phase locking. The PLF of uncorrelated and uniformly distributed phases obeys the Rayleigh distribution and approaches zero in a log-log linear manner with a slope of -0.5 when $N$ approaches infinity (data not shown). For analyses with narrow windows (see Fig. $1 A-D$ ), the temporal redundancy (correlations between nearby samples) caused by the filters is considerable, and the confidence limits were obtained by estimating the surrogate distribution of PLFs with 200 randomly selected epochs of data with an identical window size. For analyses over whole recording sessions (see Figs. $1 E, F, 2,3 A, C, 4-8$ ), the value of $\mathrm{PLF}_{\text {shuffled }}$ at $N_{\max }$ was estimated for each gradiometer and frequency band by linear regression from averaged values of $\mathrm{PLF}_{\text {shuffled }}$ at $N_{\max } / 10, N_{\max } / 32, N_{\max } / 100$, and $N_{\max } / 320$. Because the Rayleigh distribution is a function of its mean only, the extrapolated estimate of the $\operatorname{PLF}_{\text {shuffled }}\left(N_{\text {max }}\right)$ gives the confidence limits directly with the ratios $\mathrm{PLF}_{\text {real }} / \mathrm{PLF}_{\text {shuffled }}$ of 1.95 and 2.42 corresponding to $p$ values of 0.05 and 0.01 , respectively. All PLF values in this study are shown using this normalization. The Wilcoxon signed-rank test was used for the statistical evaluation of task effects. In addition, where thus stated, we used Bonferroni's correction $\left(p \rightarrow p_{\mathrm{B}}\right)$ for multiple statistical comparisons and/or exploited binomial statistics to estimate the probability $\left(p_{\text {bin }}\right)$ that the observed statistically significant effects would have occurred by chance. The binomial probability ( $p_{k \text { out of } n}$ ) of finding by chance $k$ tests significant out of $n$ possible is given by the following equation:

$$
p_{\text {bin }}=n ![k !(n-k) !]^{-1}\left(p^{k}\right)\left[(1-p)^{n-k}\right],
$$

where $p$ denotes the significance level. $p_{\text {bin }}$ used here is formulated as the probability of finding $k_{\mathrm{o}}$ or more tests significant and hence is given by the following equation:

$$
p_{\text {bin }}=\sum_{k=K_{\mathrm{o}}}^{n} \mathrm{n} ![k !(n-k) !]^{-1}\left(p^{k}\right)\left[(1-p)^{n-k}\right],
$$

where $k_{\mathrm{o}}$ denotes the observed number of tests exceeding the significance threshold $(p<0.01)$.

Quantification of CF amplitude correlations. The amplitude envelope values (obtained with Morlet wavelets) of two frequency bands were paired and sorted into 10 bins to obtain histograms of the mean amplitudes of $X$ in the 10 percentiles of amplitude $Y$, and vice versa for mean amplitudes of $Y$ as a function of the 10 percentiles of amplitude $X$. These histograms were normalized with the respective maximum variations of $X$ and $Y$ quantified with histograms of mean amplitudes of $X$ in the 10
A
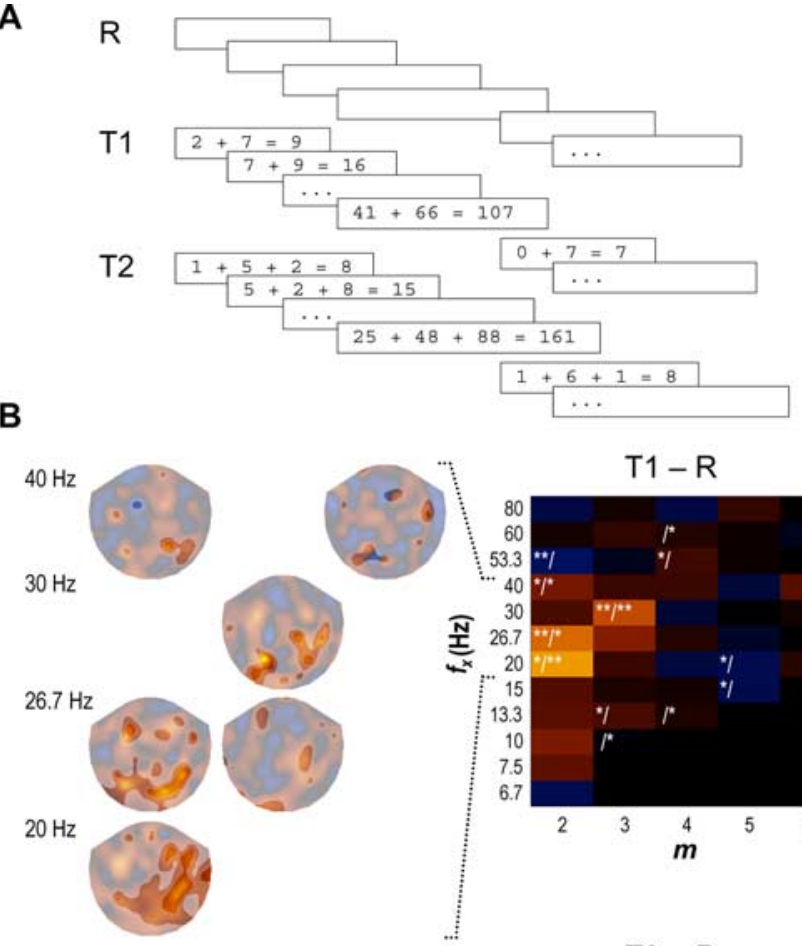

$30 \mathrm{~Hz}$
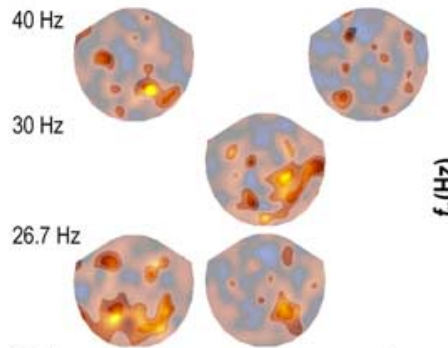

$20 \mathrm{~Hz}$

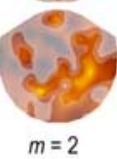

$m=3 \quad m=4$

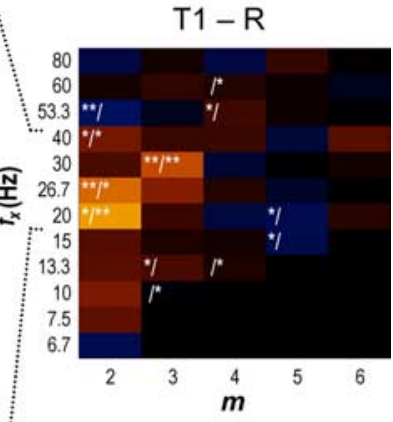

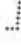
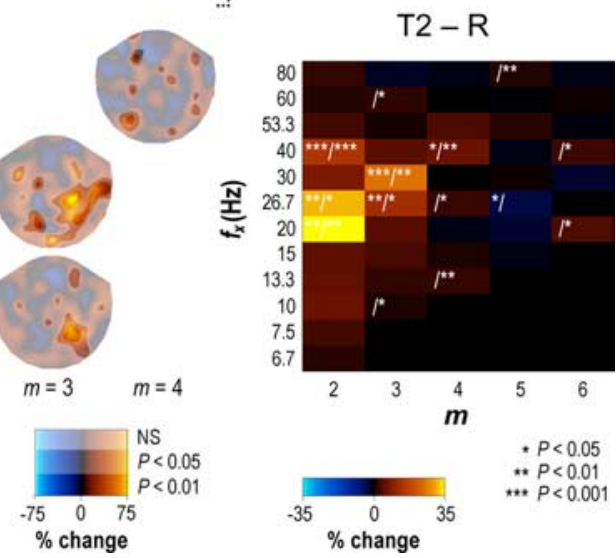

Figure 2. Mental arithmetic tasks strengthen local CF phase synchrony. $\boldsymbol{A}$, Schematic illustration of the tasks $\mathrm{R}, \mathrm{T} 1$, and $\mathrm{T} 2$ used in the study (see Materials and Methods). $\boldsymbol{B}$, Relative changes in the $\mathrm{PLF}_{1: m}$ (color scale) are averaged over gradiometers and subjects (right panels). Asterisks indicate the statistical significance of changes in the $\mathrm{PLF}_{1: m}$ (left of/in each element) and in the percentages of statistically significant channels (right of/in, as in Fig. 1E). The topographies of the most prominent changes in $\mathrm{PLF}_{1: m}$ (color scale) and their statistical significances (transparency levels) are shown in the left panels. Note that $p_{\text {bin }}$ was $<0.02$ for finding statistically significant $(p=0.01)$ task effects by chance in more than three gradiometer locations. NS, Not significant.

percentiles of amplitude $X$, and likewise for $Y$. After normalization, the sum over histogram $X(Y)$, ranging from 0 to 1 , reflects the dependency of $X$ on $Y$ and conversely for $Y(X)$. The larger of these sums for each gradiometer and $f_{x, m}$ pair was selected and averaged over gradiometers and subjects (see Fig. 3B). The advantage of this approach (over, for example, Spearman's correlation coefficient) is that it allows for the evaluation of the form of interaction and also the discovery and quantification of nonmonotonic (e.g., quadratic) interactions.

Topographical visualization of MEG data. Each MEG recording site contains two orthogonal gradiometers. For the topographical visualization of PLFs for within-gradiometer synchrony (see Figs. $1 F, 2 B$ ), we used the gradiometer with the larger PLF. Correspondingly, for the visualization of between-gradiometers synchrony (see Figs. 4, 5), we used the gradiometer pair (of the four possible combinations) with the largest 
A

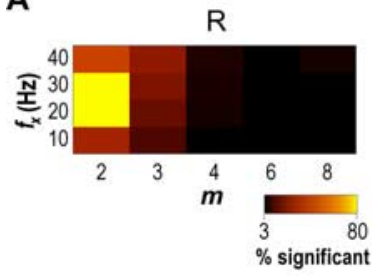

C

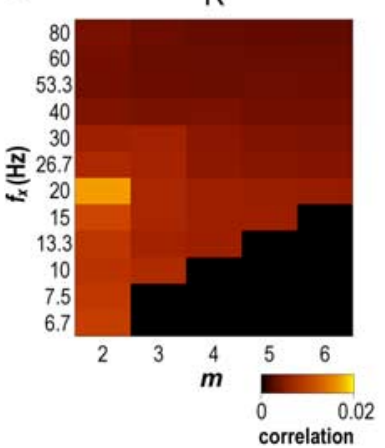

B

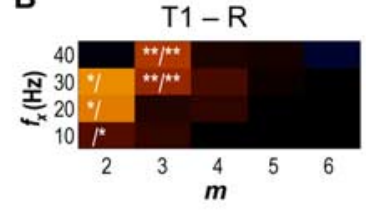

D

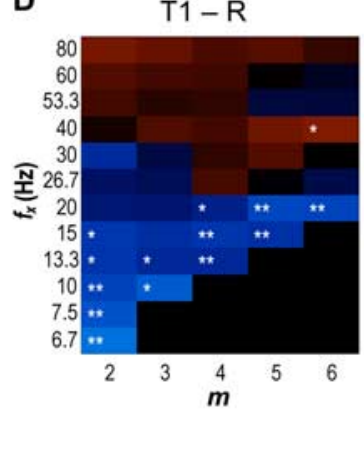

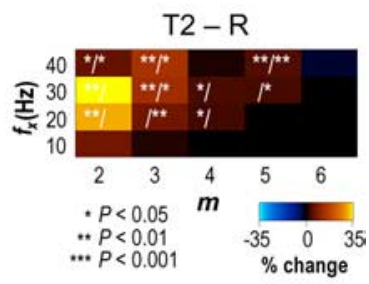

$\mathrm{T} 2-\mathrm{R}$

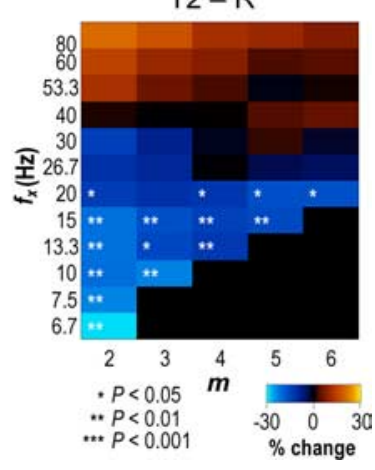

Figure 3. CF phase synchrony and $(F$ amplitude correlations. $A$, Phase synchrony $(1: m)$ mapped with broadband filtering and Hilbert transform (see Materials and Methods) over the 20 min recording sessions (compare with Fig. 1E). The color scale indicates the percentage of gradiometers detecting significant $(p<0.01)$ synchrony. $\boldsymbol{B}$, Task effects on the PLF ${ }_{1: m}$ obtained with broadband filtering and the Hilbert transform (color scale), averaged over gradiometers and subjects (compare with Fig. 2 B). Asterisks indicate the statistical significance of changes in the $\mathrm{PLF}_{1: m}$ (left to/) and in the percentages of statistically significant channels (right to/). C, CF amplitude correlations within gradiometers quantified with cross-amplitude histograms and averaged over gradiometers and subjects. $\boldsymbol{D}$, The task effects on (F amplitude correlations and the significance levels (compare with Fig. 2B).

PLF. Hence, the matrices of phase synchrony between gradiometers (see Figs. 4, 6) are reduced to a size of $102 \times 102$ from the original $204 \times 204$. To highlight the topography of medium- and long-range connections in the visualization of interareal phase synchrony during T0 (see Fig. 4) despite the exponential decrease in synchrony with increasing pair separation (data not shown), we plotted 50 gradiometer pairs for each of the 10 incremental distance scales. The topographical maps of mean interareal phase synchrony (i.e., the color maps underneath the lines linking gradiometers in Fig. 4) were obtained by averaging all PLFs for the phase synchrony of a given gradiometer with all other gradiometers. This gives an approximate measure of the inter-regional differences in large-scale phase synchrony (see Fig. 4) and the task effects thereof (see Fig. 5). Note that highly significant task effects were observed between T2 and T1, although they were not shown by this measure (compare Figs. $5 C, D$, $6 \mathrm{E}-\mathrm{H}, 8)$

\section{Results}

Cross-frequency phase synchrony among cortical oscillations Superimposed $\alpha$-frequency $(8-14 \mathrm{~Hz})$ and $\beta$-/ $\gamma$-frequency $(14-50 \mathrm{~Hz})$ band oscillations were readily observed in filtered gradiometer data (Fig. $1 A$ ) recorded during $\mathrm{R}$ (see Materials and Methods). We first addressed the presence of CF phase synchrony, which thus far has remained undiscovered in cortical activity. We obtained the continuous phase (Fig. $1 B$, red and blue traces) of the $\alpha$ and $\beta / \gamma$ oscillations using the Hilbert transform, evaluated their phase difference (Fig. $1 B$, purple trace), and computed 1: $m$ phase-locking factors in narrow sliding time windows (Fig. 1C,D) ( PLF $_{1: m}$; see Materials and Methods). We found that intermittent periods of $1: 3$ and $1: 4 \gamma-\alpha($ Fig. $1 A-D)$ as well as $1: 2$ $\beta-\alpha$ (data not shown) phase synchronies were strikingly prominent in the spontaneous activity of posterior brain areas.

To obtain the continuous phases of 3-80 Hz activities over whole recording sessions and within all MEG gradiometers, we then mapped the occurrence of CF phase synchrony by using Morlet wavelets and estimated the corresponding PLF P $m_{m}$ with $m=2-6$. Figure $1 E$ shows the percentage of gradiometers detecting statistically significant $(p<0.01)$ synchrony between oscillations at frequencies $f_{x}$ and $f_{y}$ (color scale) averaged over subjects $(N=17)$. The frequency of the faster oscillation $\left(f_{x}\right)$ is plotted on the $y$-axis, and the $m$ factor that gives the frequency of the slower oscillation $\left(f_{y}\right)\left(f_{y}=f_{x} / m\right)$ is on the $x$-axis. Because the gradiometers are the most sensitive to neuronal sources directly underneath them (Hämäläinen et al., 1993), such within-channel CF phase synchrony reflects mainly local interactions in cortical networks. During rest, we found significant 1:2 or 1:3 phase synchronies between all analyzed frequency bands (Fig. $1 E$ ). Phase synchronies (1:2) were dominated by the locking of $\beta$ - $(\sim 20 \mathrm{~Hz})$ and $\alpha$-band $(\sim 10 \mathrm{~Hz})$ oscillations widely over the cortex but predominantly over occipitoparietal and central/somatomotor regions (Fig. $1 F$, left). However, 1:3 phase synchrony was found mainly between $\gamma$-band $(\sim 30$ $\mathrm{Hz}$ ) and $\alpha$-band oscillations and mostly over the occipitoparietal and somatomotor regions (Fig. $1 F$, right).

\section{Mental arithmetic tasks enhance local CF phase synchrony}

Next, we asked whether these patterns of CF phase synchrony would be affected by mental arithmetic tasks T1 and T2 (Fig. 2A) (see Materials and Methods). Both tasks strongly enhanced local $1: 2 \gamma-\beta, 1: 2 \beta-\alpha$, and 1:3 $\gamma-\alpha$ synchronies (Fig. $2 B$ ) compared with $\mathrm{R}$. These effects were most pronounced over righthemispheric parietal and temporal/prefrontal areas but were also highly significant over corresponding left-hemispheric areas (Fig. $2 B)$. The differences in local CF phase synchrony between $\mathrm{T} 1$ and T2 were much more subtle and exhibited greater intersubject variability. Interestingly, however, T2 was associated with significantly stronger $1: 3 \quad \gamma-\alpha$ synchrony than T1 over righthemispheric parietal areas (average over nine parietal gradiometer locations: right hemisphere, $17 \%, p<0.01$; left hemisphere, $1.6 \%, p>0.8$; also see below, the $\mathrm{T} 2$ vs $\mathrm{T} 1$ data on interareal phase synchrony).

\section{The contribution of artifactual harmonics to $\mathrm{CF}$ phase synchrony}

The frequency content of oscillations with nonsinusoidal (spiky, for instance) waveforms is not limited to the band corresponding to the fundamental frequency of the oscillation. Such nonsinusoidal signals produce harmonic peaks in power spectra and $\mathrm{CF}$ phase synchrony that is artifactual in the sense that it does not reflect an interaction between two oscillations at distinct frequencies. Nonsinusoidal waveforms, in contrast, also arise when two interacting oscillations at different frequencies are actually present in the data. We addressed whether the present observations of CF synchrony can be accounted for by artifactual harmonics.

First, visual inspection of the data revealed that, during periods of prominent CF phase synchrony, the faster oscillation was also detectable in broadband-filtered data, indicating that it did not arise as a filter artifact from the slower oscillation (Fig. $1 \mathrm{~A}$ ). 
Second, we estimated CF phase synchrony over recording sessions using the Hilbert transform and temporally very narrow, broad-pass-band filters (Fig. $3 A, B$ ) (see Materials and Methods). The results were comparable with those obtained with Morlet wavelets, whereas CF phase synchrony caused by filter artifacts should have been dramatically weaker (compare Figs. $1 E, 3 A$ and Figs. $2 B, 3 B$ ). Third, we estimated the strength and form of CF amplitude correlations within gradiometers with cross-amplitude histograms (Fig. 3C) and Spearman's rank-order coefficients (data not shown). The CF amplitude correlations were, in general, extremely weak, albeit positive (Fig. 3C). If the detection of faster oscillations was strongly biased by nonsinusoidal properties of the slower oscillations, the amplitudes of faster oscillations should have been tightly linked to the amplitude of the slower oscillations. Moreover, in contrast to the band-specific enhancements of CF phase synchrony (Fig. 2B), T1 and T2 robustly weakened the amplitude correlations between all frequency pairs below the $\gamma$ band compared with $\mathrm{R}$ (Fig. 3D). This indicates that the enhancement of CF phase synchronies during T1 and T2 was not attributable to changes in $\alpha$ waveform.

Together, several lines of evidence indicate that CF phase synchronies reported in this study do indeed reflect the interactions of networks oscillating in distinct frequency bands.

\section{Interareal phase synchrony}

The data above (Figs. 1-3) showed that CF phase synchrony is present among cortical oscillations and that it is modulated by dif-

ferences in cognitive operations. To obtain a more complete picture of its characteristics, we then addressed phase synchrony between anatomically distinct areas. We quantified 1:2 and 1:3 phase synchronies of 10,20, and $30 \mathrm{~Hz}$ oscillations for all pairs of the MEG gradiometers as well as the "classical" 1:1 synchronization within these frequency bands. In rest, 1:1 phase synchrony was extremely robust for all frequency bands analyzed and highly significant for essentially every gradiometer pair (Fig. $4 A$ ). In contrast, 1:2 and especially 1:3 phase synchronies were weaker and more local (Fig. 4B). Furthermore, although 1:1 phase synchronies were rather homogeneously distributed over the cortex, 1:2 and 1:3 phase synchronies were concentrated over posterior cortical areas (Fig. 4, matrices). Such qualitative differences in the distributions of $1: 1$ and 1:2/1:3 phase synchronies suggest that the mechanisms and functions of CF and 1:1 phase synchronies are indeed distinct.

It should be noted that the magnetic field gradients produced by a focal cortical source are detected by several neighboring gradiometers that are located side by side above the source (Hämäläinen et al., 1993). Therefore, such signal spreading may contribute to observations of cross-channel synchrony between adjacent or very nearby gradiometers.
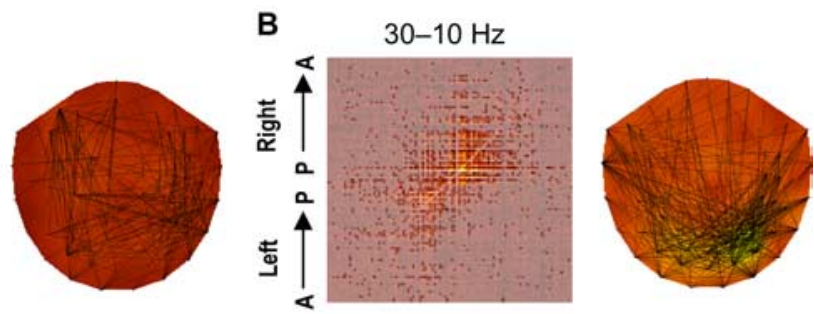

$20-10 \mathrm{~Hz}$
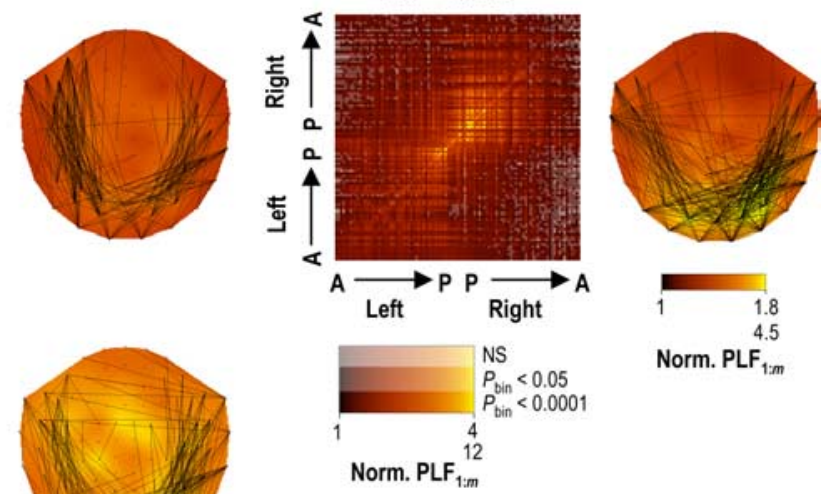

Norm. PLF

Figure 4. $\quad \boldsymbol{A}, \boldsymbol{B}$, Interareal phase synchrony within $(\boldsymbol{A})$ and between $(\boldsymbol{B}) \alpha, \beta$, and $\gamma$ oscillations in $\mathrm{R}$. The color scale of the $y$-axes. The gradiometers are ordered so that the axes run from the most anterior $(A)$ to the most posterior $(P)$ left-hemispheric eter and then conversely for the right hemispheric gradiometers. The levels of transparency indicate the binomial prob(he observed number of subjects with a statistically significant synchrony (as in Fig. 1F). The each gradiometer with all other gradiometers. The strengths of all synchronies during $R$ decayed exponentially with increasing distance (data not shown), and all synchronies were also stronger within than between the hemispheres. NS, Not significant.

\section{Task effects on interareal phase synchrony}

The execution of $\mathrm{T} 1$ and $\mathrm{T} 2$, compared with $\mathrm{R}$, enhanced interareal 1:1 phase synchronies in all frequency bands (Fig. 5A), although the strengthening of $10 \mathrm{~Hz}$ phase synchrony was clearly most pronounced and widespread (Fig. $6 A, B$ ). The $10 \mathrm{~Hz}$ phase synchrony was enhanced predominantly among posterior and anterior regions (Fig. 6A, $B$ ) and showed the strongest task effects at long distances [i.e., far from the matrix diagonal (Fig. 6A,B)]. We also plotted, as a function of gradiometer separation, the mean phase synchrony within (solid colors) and between (twocolored lines) the gradiometers in four quadrants of the MEG helmet (Fig. 7). These graphs corroborate the findings above and further highlight the central role of right-hemispheric posterior regions in large-scale $10 \mathrm{~Hz}$ synchrony (Fig. $7 \mathrm{~A}$, red lines in the $10-10 \mathrm{~Hz}$ row). A conspicuous feature of T1/T2-related $10 \mathrm{~Hz}$ phase synchrony was the marginality of task effects on synchrony within and between left-hemispheric posterior and anterior regions, despite the fact that these regions were massively synchronized with the right hemisphere (Figs. $6 \mathrm{~A}$, lower-left quadrant of the $10 \mathrm{~Hz}$ matrix, $7 A$, yellow and green lines). Phase synchronies (20 and $30 \mathrm{~Hz}$ ), on the other hand, were predominantly found within posterior and between posterior and anterior brain re- 


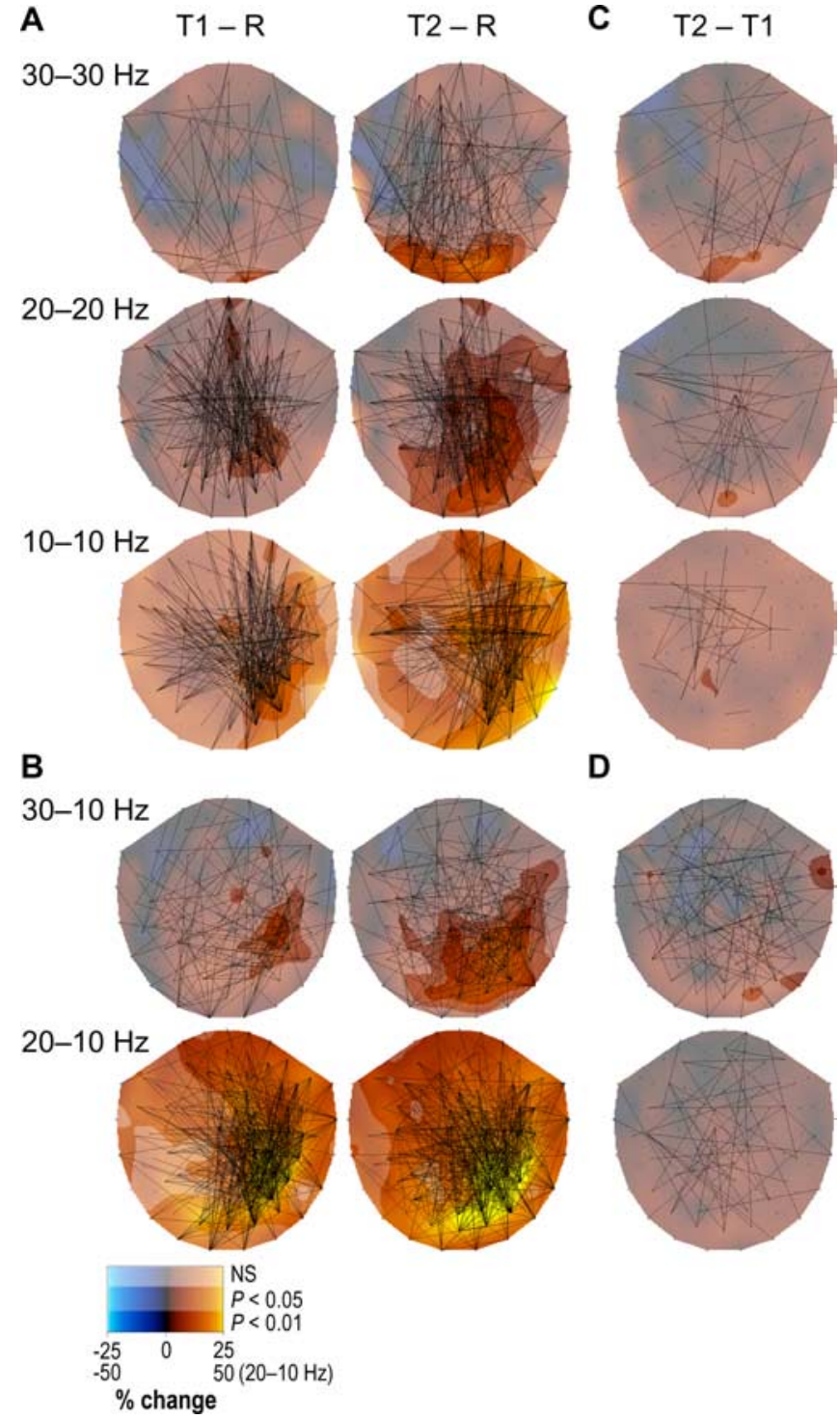

Figure 5. Task effects on interareal phase synchronies. $\boldsymbol{A}-\boldsymbol{D}$, Lines show the 500 gradiometer pairs with the most statistically significant task effects (for complete data, see Fig. 6). The underlying topographical maps show the task effects on the mean phase synchrony for each gradiometer (as in Fig. 4). The levels of transparency indicate the statistical significance of the task effects on mean phase synchrony. Note that there were also highly significant differences between $\mathrm{T} 1$ and $\mathrm{T2}(\boldsymbol{C}, \boldsymbol{D})$, although they were not significant in the topographies of mean phase synchrony (Figs. 6, 8). NS, Not significant.

gions (Fig. $5 A$ ) but much less so within anterior regions (Figs. $6 A, B$, matrix corners, $7 A$, blue and green lines) and with much less prominent right-hemispheric dominance than that observed for the $10 \mathrm{~Hz}$ phase synchrony (Fig. 6A).

Interareal 1:2 $\beta-\alpha$ and 1:3 $\gamma-\alpha$ synchronies were also considerably stronger during T1 and T2 than during R (Fig. 5B). Surprisingly, the interareal CF phase synchronies, $1: 2 \beta-\alpha$ in particular, were enhanced even more than the $1: 1$ phase synchronies (Fig. 6, compare $A, B$ with $C, D$; note the different range for $\beta-\alpha$ synchrony). Moreover, in stark contrast to the $1: 1$ phase synchronies, the CF phase synchronies were most strongly enhanced on short distances (Fig. 7B) and over the right hemispheric posterior regions (Fig. $6 C, D$ ), providing additional evidence for the fundamentally distinct characters of $1: 1$ and CF phase synchronies. The strongest long-range CF phase synchronies were found between right posterior and anterior regions as well as between right and left posterior regions (Fig. 7B, blue-red and red-yellow lines, respectively).

The differences in interareal 1:1 (Fig. 5C) and CF (Fig. 5D) synchronies between $\mathrm{T} 1$ and $\mathrm{T} 2$ were much weaker than between T1/T2 and R (Fig. 5, compare $A, B$ ). However, although the differences were relatively small, $1: 3 \quad \gamma-\alpha$ phase synchrony was clearly stronger during T2 than during T1 (Fig. 6G,H), mainly over right parietal regions (Figs. $6 \mathrm{G}, \mathrm{H}, 8 \mathrm{~B}$, red line). In contrast, the massive 1:2 $\beta-\alpha$ synchrony showed only a small difference between $\mathrm{T} 1$ and $\mathrm{T} 2$, suggesting that the functional roles of these $1: 2$ and 1:3 phase synchronies are distinct. In addition, 1:1 phase synchronies were significantly stronger in T2 than in T1 (Fig. $6 E, F$ ) but were enhanced less than the $1: 3 \gamma-\alpha$ synchrony (Fig. 8 , compare $A, B)$. Interestingly, $10 \mathrm{~Hz} 1: 1$ phase synchrony was enhanced within the left hemisphere and bilaterally within the anterior regions, apparently linking and recruiting specifically those areas that were the least involved in the task execution in T1. Because T1 and T2 differed essentially only in the number of simultaneously memorized object representations, these data strongly suggest that $1: 3 \gamma-\alpha$ phase synchrony has a role in the neuronal mechanisms of working memory maintenance.

\section{Task effects on oscillation amplitudes}

To relate the data obtained with the present tasks to previous studies using working memory paradigms, we evaluated the oscillation amplitudes with Morlet wavelets for all frequency bands in Figures 1-3. The results showed that amplitudes of $\alpha$ (Bastiaansen et al., 2002; Halgren et al., 2002; Jensen et al., 2002) and $\beta / \gamma$ (Tallon-Baudry et al., 1998; Pesaran et al., 2002; Howard et al., 2003) bands were slightly but significantly increased in T1 and T2 compared with R (Fig. 9). The amplitude enhancements were mainly observed over occipitoparietal and temporal/prefrontal areas. Interestingly, the amplitudes of $\theta$-band (3-8 Hz) oscillations were enhanced over prefrontal regions (Gevins et al., 1997; Howard et al., 2003) but were suppressed widely over the posterior regions (cf. Bastiaansen et al., 2002).

\section{Discussion}

Although the well established "temporal correlation hypothesis" (Singer, 1999) underscoring within-frequency synchrony has been successful in explaining neuronal integration at the level of feature binding, the demand for CF interactions in higher-level neuronal processing has also been recognized (Lisman and Idiart, 1995; Engel and Singer, 2001; Varela et al., 2001; VanRullen and Koch, 2003). Relatively few studies have thus far investigated nonlinear (CF) phase coupling (Jeffrey and Chamoun, 1994; Shils et al., 1996; Schanze and Eckhorn, 1997; von Stein et al., 2000; Schack et al., 2002) or nested oscillations (Chrobak and Buzsaki, 1998; Schack et al., 2002; Bruns and Eckhorn, 2004; Vanhatalo et al., 2004). We have shown here that, among cortical oscillations, CF phase interactions are also found in the form of 1:m-type CF phase synchrony. CF phase synchrony was a salient characteristic of ongoing activity in the human cortex, and it was strongly influenced by differences in cognitive task demands, supporting its role in higher-level neuronal processing. Although future studies will need to pinpoint its specific functional roles, several lines of evidence support an idea that CF phase synchrony is a plausible mechanism for the integration of spectrally distributed processing. Unlike CF amplitude correlations and nested oscillations, it provides a way to integrate spectrally distributed processing with the temporal precision of the faster oscillation. 
A

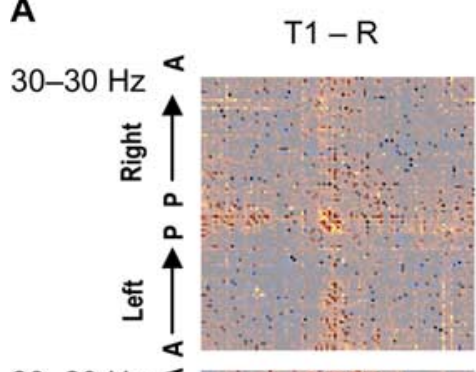

20-20 Hz

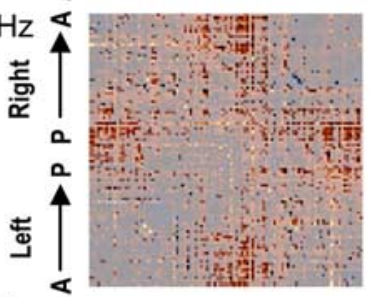

10-10 Hz

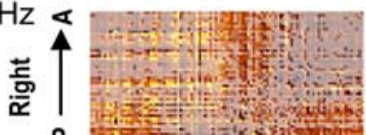

a.

实
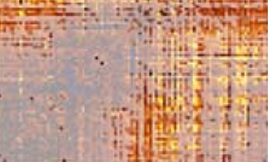

C

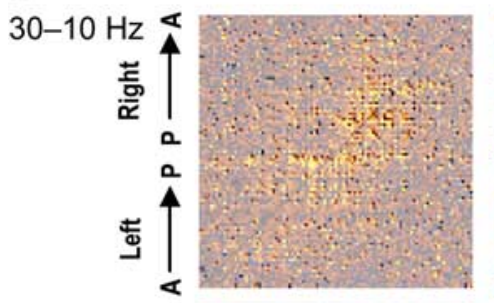

20-10 Hz

8

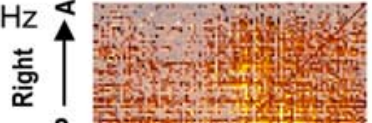

a
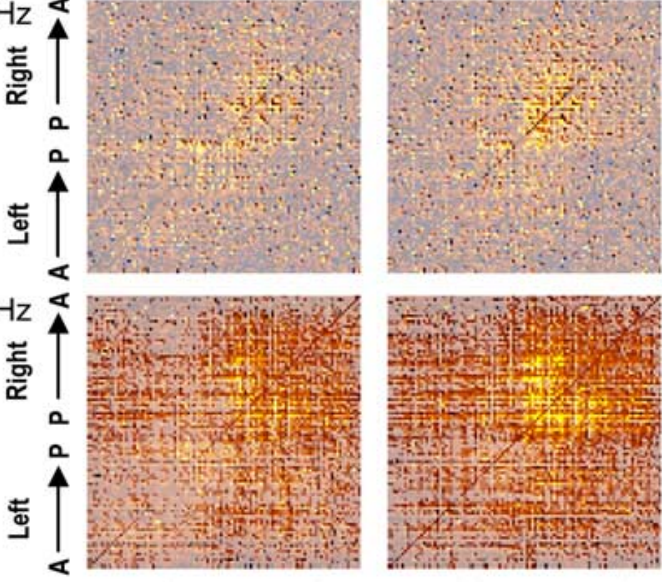

D

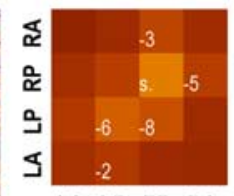

LA LP RP RA
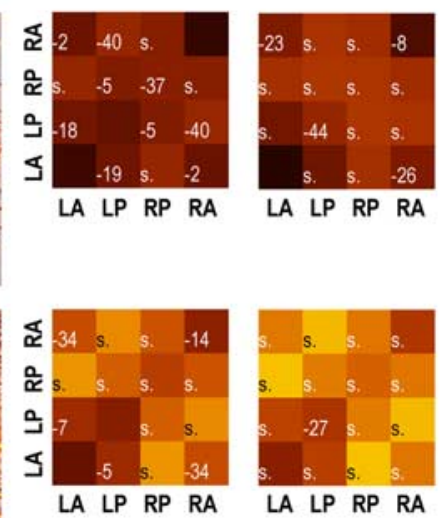

E
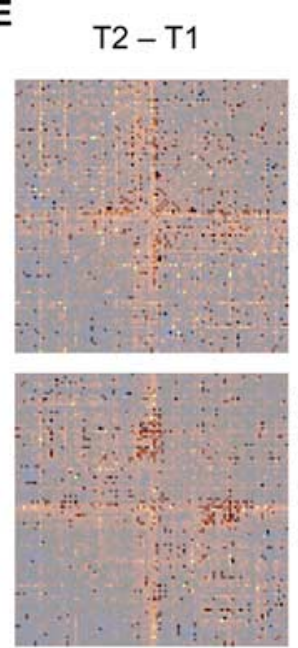

文

\&

a

5

LA LP RP RA
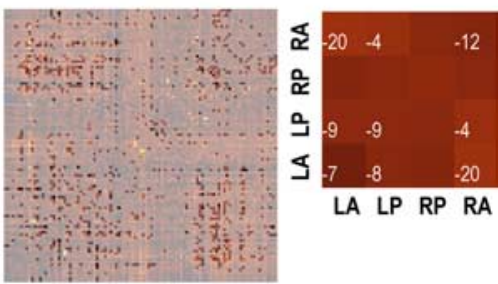

LA LP RP RA
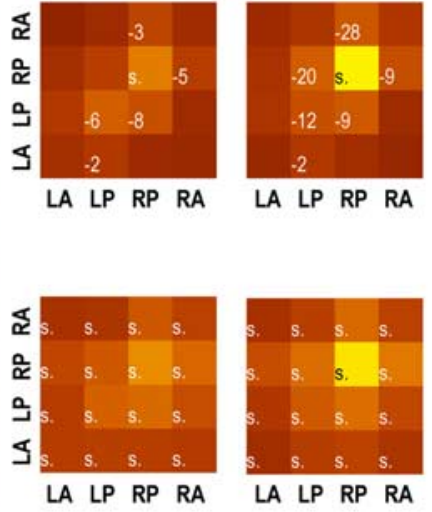

G

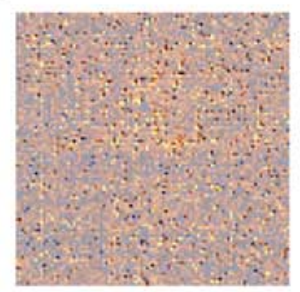

H
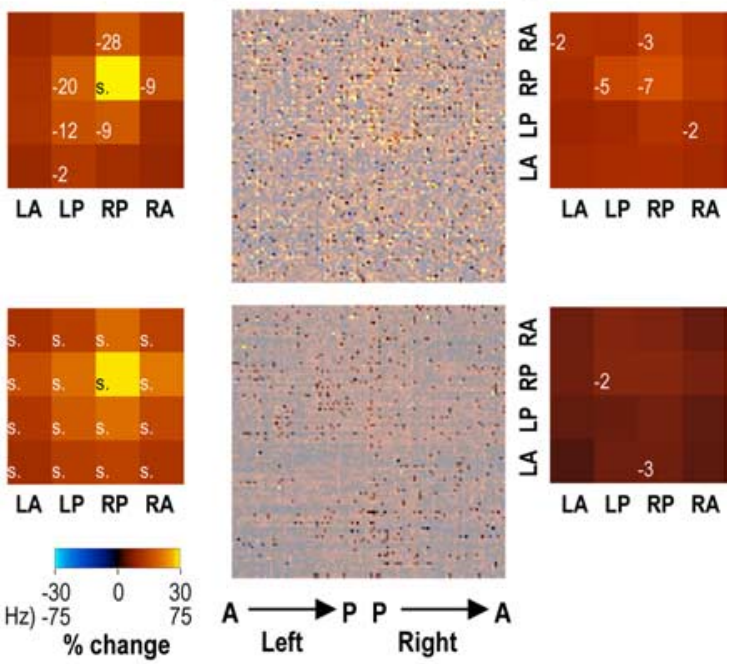
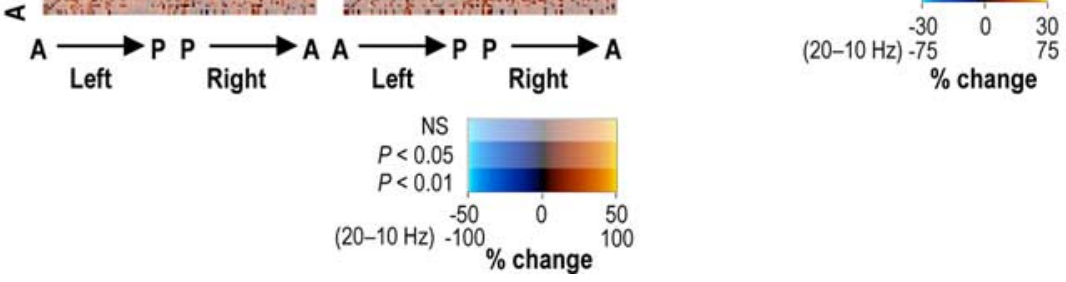

Figure 6. Task effects on interareal phase synchronies. $A, C, E, G$, Matrices showing the task effects on $\mathrm{PLF}_{1: m}$ for all gradiometer pairs are ordered as in Figure 4. The color scale indicates the magnitude, and the levels of transparency indicate the statistical significance of task effects. $B, D, F, H$, The contribution of spurious significances to data in $A, C, E$, and $G$. Binomial statistics were used to evaluate the probability of finding by chance the number of statistically significant gradiometer pairs in the corresponding matrices. The numeric values indicate the exponent $a$ for Bonferronicorrected (16 matrix elements) $p_{\mathrm{B}, \text { bin }}<10^{a}$. (a values more than -2 were not considered significant; $a$ values less than -53 are marked with s.) The underlying color map shows the magnitude of the task effect, averaged over the respective gradiometer pairs. A, Anterior; $P$, posterior; LA, left anterior; LP, left posterior; RA, right anterior; RP, right posterior; NS, Not significant.

\section{The enhancement of CF phase synchrony by} cognitive processes

CF phase synchrony was robust and present during each task among all frequency bands analyzed. However, mental calculation and working memory demands of $\mathrm{T} 1$ and $\mathrm{T} 2$ enhanced specifically the 1:2-1:4 phase synchronies among $\alpha, \beta$, and $\gamma$ oscillations. The enhancements of the 1:1 phase synchrony, the oscillation amplitudes, and the distinct spatial patterns of 1:1 synchrony of these oscillations, imply, in good agreement with other evidence (Singer, 1999; TallonBaudry and Bertrand, 1999; von Stein et al., 2000; Varela et al., 2001; Howard et al., 2003), that $\alpha, \beta$, and $\gamma$ oscillations are all involved in the performance of $\mathrm{T} 1$ and $\mathrm{T} 2$ but with distinct functional roles. Thus, CF phase synchrony among these oscillations is well posed to mediate the integration of this spectrally distributed processing into 
A
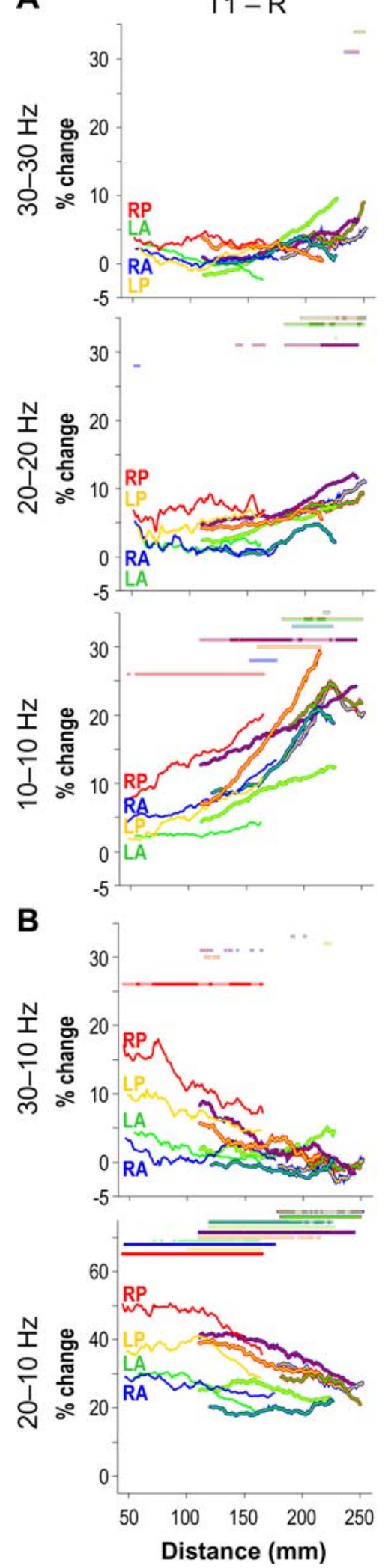

$\mathrm{T} 2-\mathrm{R}$
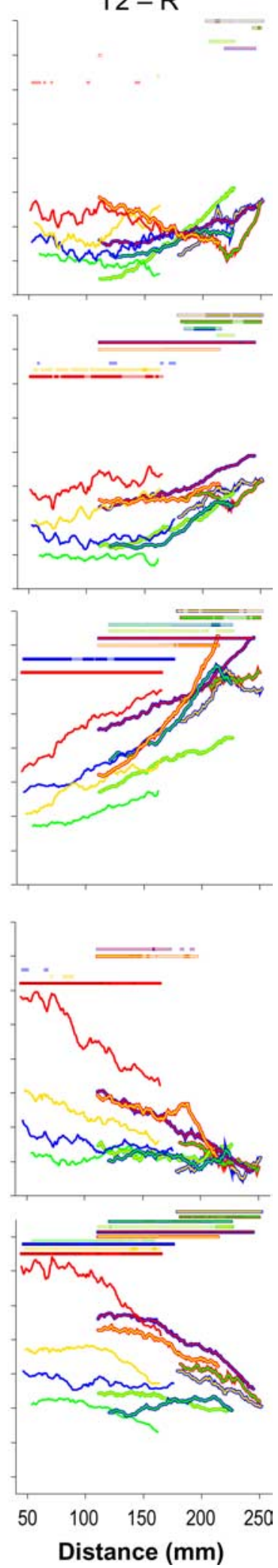

Figure 7. Task effects on interareal phase synchronies as a function of gradiometer pair separation; T1/T2 versus R. To corroborate and extend the findings in Figures 5 and 6 , we quantified the task effects on phase synchrony within and between the gradiometer groups in the four quadrants of the MEG helmet. The lines show the sliding average PLF $P_{1: m}$ ( $y$-axis) of 125 gradiometer pairs as a function of their mean pair separation (see $x$-axis). $\boldsymbol{A}, \boldsymbol{B}$, The lines are colored so that the right posterior (RP) quadrant is marked with red, the left posterior (LP) quadrant is marked with yellow, the right anterior (RA) quadrant is marked with blue, and the

A
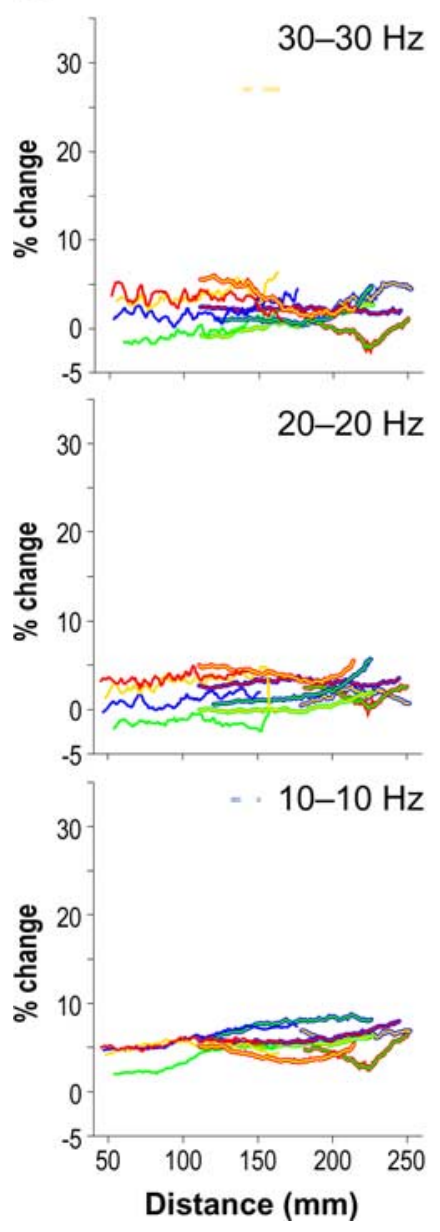

B
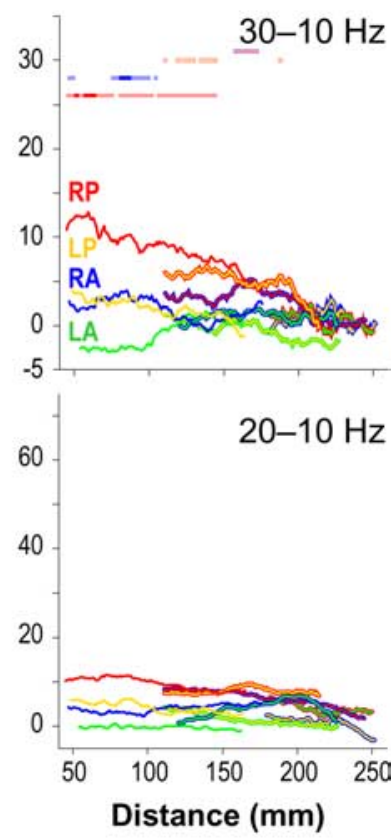

Figure 8. Task effects on interareal phase synchronies as a function of gradiometer pair separation; T1 versus T2. Line colors, scales, and statistics are as in Figure 7.A, Phase synchronies (1:1). B, Phase synchronies (1:2 and 1:3). LA, Left anterior; LP, left posterior; RA, right anterior; $\mathrm{RP}$, right posterior.

the cognitive operations required by mental calculations and working memory.

The role of 1:3 $\gamma-\alpha$ phase synchrony in working memory The mental arithmetic tasks in this study affected the amplitude, 1:1 phase synchrony, and hemispheric lateralization of ongoing neuronal activity in a manner very similar to that observed previously in working memory tasks, which is well in line with the notion that the principal task load during easy mental calculation is on the working memory system (Houdé and Tzourio-Mazoyer, 2003). Nevertheless, the comparison of task T1/T2 and rest does not allow the dissection of working memory-related activities from those involved in, for example, the mental calculation, but reveals the overall differences between minimal and nearmaximal cognitive load. However, the comparison of tasks T1 and $\mathrm{T} 2$ reveals specifically the components sensitive to working

left anterior (LA) quadrant is marked with green. Lines with a single color show the task effects on synchrony within the quadrant corresponding to that color. Lines with two colors show the task effects on synchrony between the two quadrants indicated by the colors. The horizontal bars indicate the significance level of the task effect; light color, $p<0.05$; medium color, $p<$ 0.01 ; dark color, $p<0.001$. Note that $p<0.01$ corresponds to $p_{\mathrm{B}}<0.05$ after Bonferroni's correction with the number of independent averaging windows (5). 
memory load. It is therefore intriguing to find that $1: 3 \quad \gamma-\alpha$ phase synchrony was moderately but highly significantly stronger during $\mathrm{T} 2$ than during $\mathrm{T} 1$. This result suggests that $1: 3$ synchronized $\gamma$ and $\alpha$ oscillations may play a role in the neuronal mechanisms of working memory. Taking the proposed "representational" (Singer, 1999; Tallon-Baudry and Bertrand, 1999) and attentional (von Stein et al., 2000; VanRullen and Koch, 2003) roles of $\beta / \gamma$ and $\alpha$ oscillations, the results suggest that such 1:3 synchronization of these oscillations may mediate the contents-to-context binding of neuronal object representations into working memory and/or the multiplexing of these object representations, which is required when multiple representations must be kept active simultaneously.

\section{Parietal focus of CF phase synchrony}

$\beta-\alpha$ and $\gamma-\alpha$ phase synchronies were strongest over the right hemispheric parietal regions, as were their task-related enhancements. At the same time, the corresponding 1:1 synchronies were widely distributed over the cortex. In addition to the fact that these results support the differential origin of CF phase and withinfrequency synchronies, they also support the notion that CF phase synchronies are related to attentional and working memory functions. The right-hemispheric parietal regions have been shown to play a critical role in attention and working memory by imaging studies (Corbetta et al., 1998; Kastner and Ungerleider, 2000; Awh and Jonides, 2001; Linden et al., 2003) and by the diversity of attentional deficits caused by parietal damage (Friedman-Hill et al., 1995; Driver and Vuilleumier, 2001). In addition, the brain areas specifically responsible for multiplexing functions (Friedman-Hill et al., 1995) are located, at least partly, in the parietal regions, as suggested again both by imaging studies localizing cortical activity correlated with working memory capacity (Linden et al., 2003) and by findings that damage in parietal regions can result in an inability to perceive multiple objects simultaneously (simultagnosia) (Friedman-Hill et al., 1995).

\section{Putative cellular-level mechanisms involved in CF phase synchrony}

The concept of "dynamic binding" posits that the postsynaptic impact of "bound" presynaptic inputs is greater than the impact of "unbound" ones (Singer, 1999). Although excitatory inputs synchronized in a 1:1 manner have better chances of evoking action potentials in downstream target neurons than asynchronous inputs (Konig et al., 1996; Singer, 1999), little is known about the functional significance of $1: m(m>1)$ synchronization in neuronal signaling.

Interestingly, a type of neocortical layer 5 (L5) pyramidal cell with dendrites spanning all cortical layers has characteristics that are relevant in this context. The main excitatory connections to proximal and basal dendrites of these cells originate from specific thalamic nuclei (Jones, 2002), from local layer 4 spiny stellate cells, and, overall, from cortical areas at the same level or lower in hierarchy (Felleman and Van Essen, 1991). In contrast, inputs to distal apical dendrites primarily arise from diverse nonspecific thalamic regions (Jones, 2002) and from cortical areas higher in hierarchy (Felleman and Van Essen, 1991; Cauller et al., 1998). L5 pyramidal cells thus receive bottom-up and top-down signals in distinct integrative compartments, and both proximal (bottomup) and distal (top-down) inputs alone, if sufficiently strong, can evoke action potentials or burst firing (Helmchen et al., 1999; Larkum et al., 1999; Williams and Stuart, 1999; Larkum and Zhu, 2002). However, burst firing is facilitated if the proximal and distal inputs are temporally correlated with a precision of $\sim 5 \mathrm{~ms}$ (Larkum et al., 1999). Because bursts have a much stronger impact on postsynaptic neurons than single action potentials (Lisman, 1997; Williams and Stuart, 1999), L5 pyramidal cells can effectively signal the coincidence of bottom-up and topdown signals and thus are able to mediate cross-hierarchical integration.

Although the feedforward/bottom-up inputs to L5 pyramidal cells often involve $\gamma$-band rhythmicity (Steriade et al., 1996; Castelo-Branco et al., 1998; Singer, 1999; von Stein et al., 2000), the feedback/top-down signals are more likely to have periodicities in the $\alpha$ band (von Stein et al., 2000; Mima et al., 2001; 
Halgren et al., 2002; Yamagishi et al., 2003). L5 pyramidal cells can also entrain each other (Silva et al., 1991) as well as their subcortical targets in the brainstem (Nicolelis et al., 1995) and superior colliculus (Brecht et al., 1998) into $\sim 10 \mathrm{~Hz}$ oscillations. Moreover, the regenerative $\mathrm{Ca}^{2+}$ action potentials underlying distal dendritic excitation (Helmchen et al., 1999; Larkum et al., 1999; Larkum and Zhu, 2002) cannot occur at frequencies more than $\sim 10 \mathrm{~Hz}$ (Larkum and Zhu, 2002). The narrowness of the correlation window (Larkum et al., 1999) indicates that oscillatory proximal and distal inputs in $20-40$ and $<10 \mathrm{~Hz}$ bands, respectively, can readily induce burst firing only when they are phase synchronized in a 1:m manner. This implies that the L5 pyramidal neurons can function as "read-out devices" for $1: m$ phase synchrony. Moreover, this kind of mechanism would also enable an unambiguous and rapid attentional selection of target representations from among other intermittent assemblies. Therefore, it is of interest to note that many of the subcortical targets of L5 pyramidal cells, such as the pulvinar and superior colliculus, have a critical role in attention and visual orienting (Karnath et al., 2002).

Another type of neuron that deserves attention in this context is fast rhythmic-bursting (FRB) cells. In a manner akin to L5 pyramidal neurons, they can switch from single-spiking to burstfiring mode, in which they show very fast $(300-600 \mathrm{~Hz})$ bursts at $\gamma(30-40 \mathrm{~Hz})$ frequencies (Steriade et al., 1998, 2004). Notably, FRB cells, located in all cortical layers from 2 to 6 , are key elements in the reciprocal thalamocortical loops that are involved in the generation of $\beta$-/ $\gamma$-frequency band oscillations (Steriade et al., 1996, 1998).

\section{Conclusion}

We propose that cross-frequency phase synchrony mediates cross-hierarchical and cross-functional integration essential for unified cognitive operations by enabling the integration of spectrally distributed neuronal processing.

\section{References}

Awh E, Jonides J (2001) Overlapping mechanisms of attention and working memory. Trends Cogn Sci 5:119-126.

Bastiaansen MC, Posthuma D, Groot PF, de Geus EJ (2002) Event-related alpha and theta responses in a visuo-spatial working memory task. Clin Neurophysiol 113:1882-1893.

Brecht M, Singer W, Engel AK (1998) Correlation analysis of corticotectal interactions in the cat visual system. J Neurophysiol 79:2394-2407.

Bruns A, Eckhorn R (2004) Task-related coupling from high- to lowfrequency signals among visual cortical areas in human subdural recordings. Int J Psychophysiol 51:97-116.

Carlson S, Martinkauppi S, Rama P, Salli E, Korvenoja A, Aronen HJ (1998) Distribution of cortical activation during visuospatial n-back tasks as revealed by functional magnetic resonance imaging. Cereb Cortex 8:743-752.

Castelo-Branco M, Neuenschwander S, Singer W (1998) Synchronization of visual responses between the cortex, lateral geniculate nucleus, and retina in the anesthetized cat. J Neurosci 18:6395-6410.

Cauller LJ, Clancy B, Connors BW (1998) Backward cortical projections to primary somatosensory cortex in rats extend long horizontal axons in layer I. J Comp Neurol 390:297-310.

Chrobak JJ, Buzsaki G (1998) Gamma oscillations in the entorhinal cortex of the freely behaving rat. J Neurosci 18:388-398.

Corbetta M, Akbudak E, Conturo TE, Snyder AZ, Ollinger JM, Drury HA, Linenweber JM, Petersen SE, Raichle ME, Van Essen DC, Shulman GL (1998) A common network of functional areas for attention and eye movements. Neuron 21:761-773.

Driver J, Vuilleumier P (2001) Perceptual awareness and its loss in unilateral neglect and extinction. Cognition 79:39-88.

Dumermuth G, Huber PJ, Kleiner B, Gasser T (1971) Analysis of interrela- tions between frequency bands of EEG by means of bispectrum - preliminary study. Electroencephalogr Clin Neurophysiol 31:137-148.

Engel AK, Singer W (2001) Temporal binding and the neural correlates of sensory awareness. Trends Cogn Sci 5:16-25.

Fanselow EE, Sameshima K, Baccala LA, Nicolelis MA (2001) Thalamic bursting in rats during different awake behavioral states. Proc Natl Acad Sci USA 98:15330-15335.

Felleman DJ, Van Essen DC (1991) Distributed hierarchical processing in the primate cerebral cortex. Cereb Cortex 1:1-47.

Friedman-Hill SR, Robertson LC, Treisman A (1995) Parietal contributions to visual feature binding: evidence from a patient with bilateral lesions. Science 269:853-855.

Gevins A, Smith ME, McEvoy L, Yu D (1997) High-resolution EEG mapping of cortical activation related to working memory: effects of task difficulty, type of processing, and practice. Cereb Cortex 7:374-385.

Gray CM, Konig P, Engel AK, Singer W (1989) Oscillatory responses in cat visual cortex exhibit inter-columnar synchronization which reflects global stimulus properties. Nature 338:334-337.

Halgren E, Boujon C, Clarke J, Wang C, Chauvel P (2002) Rapid distributed fronto-parieto-occipital processing stages during working memory in humans. Cereb Cortex 12:710-728.

Hochstein S, Ahissar M (2002) View from the top: hierarchies and reverse hierarchies in the visual system. Neuron 36:791-804.

Houdé O, Tzourio-Mazoyer N (2003) Neural foundations of logical and mathematical cognition. Nat Rev Neurosci 4:507-514.

Howard MW, Rizzuto DS, Caplan JB, Madsen JR, Lisman J, AschenbrennerScheibe R, Schulze-Bonhage A, Kahana MJ (2003) Gamma oscillations correlate with working memory load in humans. Cereb Cortex 13:1369-1374.

Hämäläinen M, Hari R, Ilmoniemi RJ, Knuutila J, Lounasmaa OV (1993) Magnetoencephalography - theory, instrumentation, and applications to noninvasive studies of the working human brain. Rev Mod Phys 65:413-497.

Helmchen F, Svoboda K, Denk W, Tank DW (1999) In vivo dendritic calcium dynamics in deep-layer cortical pyramidal neurons. Nat Neurosci 2:989-996.

Jeffrey CS, Chamoun NG (1994) An introduction to bispectral analysis for the electroencephalogram. J Clin Monit 10:392-404.

Jensen O, Gelfand J, Kounios J, Lisman JE (2002) Oscillations in the alpha band $(9-12 \mathrm{~Hz})$ increase with memory load during retention in a shortterm memory task. Cereb Cortex 12:877-882.

Jones EG (2002) Thalamic circuitry and thalamocortical synchrony. Philos Trans R Soc Lond B Biol Sci 357:1659-1673.

Karnath HO, Himmelbach M, Rorden C (2002) The subcortical anatomy of human spatial neglect: putamen, caudate nucleus and pulvinar. Brain 125:350-360.

Kastner S, Ungerleider LG (2000) Mechanisms of visual attention in the human cortex. Annu Rev Neurosci 23:315-341.

Konig P, Engel AK, Singer W (1996) Integrator or coincidence detector? The role of the cortical neuron revisited. Trends Neurosci 4:130-137.

Lachaux JP, Rodriguez E, Martinerie J, Varela FJ (1999) Measuring phase synchrony in brain signals. Hum Brain Mapp 8:194-208.

Larkum ME, Zhu JJ (2002) Signaling of layer 1 and whisker-evoked $\mathrm{Ca}^{2+}$ and $\mathrm{Na}^{+}$action potentials in distal and terminal dendrites of rat neocortical pyramidal neurons in vitro and in vivo. J Neurosci 22:6991-7005.

Larkum ME, Zhu JJ, Sakmann B (1999) A new cellular mechanism for coupling inputs arriving at different cortical layers. Nature 398:338-341.

Linden DE, Bittner RA, Muckli L, Waltz, JA, Kriegeskorte, N, Goebel R, Singer W, Munk MH (2003) Cortical capacity constraints for visual working memory: dissociation of fMRI load effects in a fronto-parietal network. NeuroImage 20:1518-1530.

Lisman JE (1997) Bursts as a unit of neural information: making unreliable synapses reliable. Trends Neurosci 20:38-43.

Lisman JE, Idiart MA (1995) Storage of $7+/-2$ short-term memories in oscillatory subcycles. Science 267:1512-1515.

Llinás R, Ribary U, Contreras D, Pedroarena C (1998) The neuronal basis for consciousness. Philos Trans R Soc Lond B Biol Sci 353:1841-1849.

Luck SJ, Vogel EK (1997) The capacity of visual working memory for features and conjunctions. Nature 390:279-281.

Mima T, Oluwatimilehin T, Hiraoka T, Hallett M (2001) Transient interhemispheric neuronal synchrony correlates with object recognition. J Neurosci 21:3942-3948.

Nicolelis MA, Baccaca LA, Lin RC, Chapin JK (1995) Sensorimotor encod- 
ing by synchronous neural ensemble activity at multiple levels of the somatosensory system. Science 268:1353-1358.

Pesaran B, Pezaris JS, Sahani M, Mitra PP, Andersen RA (2002) Temporal structure in neuronal activity during working memory in macaque parietal cortex. Nat Neurosci 5:805-811.

Raghavachari S, Kahana MJ, Rizzuto DS, Caplan JB, Kirschen MP, Bourgeois B, Madsen JR, Lisman JE (2001) Gating of human theta oscillations by a working memory task. J Neurosci 21:3175-3183.

Schack B, Vath N, Petsche H, Geissler HG, Moller E (2002) Phase-coupling of theta-gamma EEG rhythms during short-term memory processing. Int J Psychophysiol 44:143-163.

Schanze T, Eckhorn R (1997) Phase correlation among rhythms present at different frequencies: spectral methods, application to microelectrode recordings from visual cortex and functional implications. Int J Psychophysiol 26:171-189.

Shils JL, Litt M, Skolnick BE, Stecker MM (1996) Bispectral analysis of visual interactions in humans. Electroencephalogr Clin Neurophysiol 98:113-125.

Silva LR, Amitai Y, Connors BW (1991) A comparison of synapses onto the somata of intrinsically bursting and regular spiking neurons in layer $\mathrm{V}$ of rat SmI cortex. Science 251:432-435.

Singer W (1999) Neuronal synchrony: a versatile code for the definition of relations? Neuron 24:49-65.

Steriade M (2004) Neocortical cell classes are flexible entities. Nat Rev Neurosci 5:121-134.

Steriade M, Amzica F, Contreras D (1996) Synchronization of fast (30-40 $\mathrm{Hz}$ ) spontaneous cortical rhythms during brain activation. J Neurosci 16:392-417.

Steriade M, Timofeev I, Dürmüller N, Grenier F (1998) Dynamic properties of corticothalamic neurons and local cortical interneurons generating fast rhythmic $(30-40 \mathrm{~Hz})$ spike bursts. J Neurophysiol 79:483-490.

Tallon-Baudry C, Bertrand O (1999) Oscillatory gamma activity in humans and its role in object representation. Trends Cogn Sci 3:151-162.
Tallon-Baudry C, Bertrand, O Peronnet F, Pernier J (1998) Induced $\gamma$-band activity during the delay of a visual short-term memory task in humans. J Neurosci 18:4244-4254.

Tallon-Baudry C, Bertrand O, Fischer C (2001) Oscillatory synchrony between human extrastriate areas during visual short-term memory maintenance. J Neurosci 21:RC177(1-5).

Tass P, Rosenblum MG, Weule J, Kurths J, Pikovsky A, Volkmann J, Schnitzler A, Freund H-J (1998) Detection of n:m phase locking from noisy data: application to magnetoencephalography. Phys Rev Lett 81:3291-3294.

Tass PA, Fieseler T, Dammers J, Dolan K, Morosan P, Majtanik M, Boers F, Muren A, Zilles K, Fink GR (2003) Synchronization tomography: a method for three-dimensional localization of phase synchronized neuronal populations in the human brain using magnetoencephalography. Phys Rev Lett 90:088101-1-088101-4.

Vanhatalo S, Palva JM, Holmes MD, Miller JW, Voipio J, Kaila K (2004) Infraslow oscillations modulate excitability and interictal epileptic activity in the human cortex during sleep. Proc Natl Acad Sci USA 101:5053-5057.

VanRullen R, Koch C (2003) Is perception discrete or continuous? Trends Cogn Sci 7:207-213.

Varela F, Lachaux J-P, Rodriguez E, Martinerie J (2001) The brainweb: phase synchronization and large-scale integration. Nat Rev Neurosci 2:229-239.

von Stein A, Chiang C, Konig P (2000) Top-down processing mediated by interareal synchronization. Proc Natl Acad Sci USA 97:14748-14753.

Williams SR, Stuart GJ (1999) Mechanisms and consequences of action potential burst firing in rat neocortical pyramidal neurons. J Physiol (Lond) 521:467-482.

Yamagishi N, Callan DE, Goda N, Anderson SJ, Yoshida Y, Kawato M (2003) Attentional modulation of oscillatory activity in human visual cortex. NeuroImage 20:98-113. 